\title{
Performance and Implementation Evaluation of TR PAPR Reduction Methods for DVB-T2
}

\author{
Mohamad Mroué, ${ }^{1}$ Amor Nafkha, ${ }^{1}$ Jacques Palicot, ${ }^{1}$ Benjamin Gavalda, ${ }^{2}$ and Nelly Dagorne ${ }^{2}$ \\ ${ }^{1}$ SUPELEC-IETR, avenue de la Boulaie CS 47601, 35576 Cesson Sévigné Cedex, France \\ ${ }^{2}$ ENENSYS Technologies, 80 avenue des Buttes de Coesmes, 35700 Rennes, France
}

Correspondence should be addressed to Mohamad Mroué, mkmroue@hotmail.com

Received 15 April 2010; Accepted 26 August 2010

Academic Editor: Jaime Lloret

Copyright (C) 2010 Mohamad Mroué et al. This is an open access article distributed under the Creative Commons Attribution License, which permits unrestricted use, distribution, and reproduction in any medium, provided the original work is properly cited.

High Peak to Average Power Ratio (PAPR) is a critical issue in multicarrier communication systems using Orthogonal Frequency Division Multiplexing (OFDM), as in the Second Generation Terrestrial Digital Video Broadcasting (DVB-T2) system. This problem can result in large performance degradation due to the nonlinearity of the High Power Amplifier (HPA) or in its low power efficiency. In this paper, we evaluate the performance of different Tone Reservation-based techniques for PAPR reduction in DVB-T2 context. Also, we propose an iterative TR-based technique called "One Kernel One Peak" (OKOP). Simulation results and performance comparison of these techniques in terms of gain in PAPR reduction, mean power variation, and complexity will be given. Finally, we describe the implementation of a PAPR reduction algorithm in the DVB-T2 modulator.

\section{Introduction}

The performance of high data rate systems is significantly limited by the multipath interference that occurs in the radio channel environment. As an attractive technique in mitigating the multipath interference, Orthogonal Frequency Division Multiplexing (OFDM) has been widely applied in various broadcasting systems such as, the Digital Video Broadcasting (DVB) systems. Despite its competitive attributes, OFDM signals are characterized by very high Peak-to-Average Power Ratio (PAPR) levels. This characteristic leads the OFDM signals to be very sensitive to nonlinearities of analogue components of the transceiver, in particular those of the High Power Amplifier (HPA) at the emission.

An HPA is conceived to operate in its saturation zone which corresponds to its high efficiency region. However, in this zone, the HPA has a severe nonlinear behaviour. These nonlinearities are sources of $I n$-Band (IB) distortions which can both degrade the link performance in term of Bit Error Rate (BER) and also cause significant Out-Of-Band (OOB) interference products that make it harder for the operator to comply with stringent spectral masks. The simplest solution to this problem is to operate the HPA in the linear region by allowing a large enough amplifier back-off. However, this approach degrades the power efficiency of the system and often leads to unacceptable cost-efficiency conditions in the overall system. For all these reasons, reducing the PAPR of OFDM signals is increasingly being considered to be very important in maintaining the cost-effectiveness advantages of OFDM in practical systems, especially as new systems, such as DVB-T2, are being specified with large number of subcarriers (up to 32768 subcarriers and 256QAM modulation for DVB-T2 system [1]).

Many methods have been proposed to mitigate the OFDM PAPR by acting on the signal itself $[2,3]$. The simplest ones use clipping and filtering techniques $[4,5]$. However, these methods may lead to BER increase of the system since clipping is a nonlinear process [6]. Alternative methods are based on coding [7, 8] and others on Multiple Signal Representation (MSR) techniques: Partial Transmit Sequence (PTS) [9], Selective Mapping (SLM) [10], and Interleaving [11]. The main drawback of these methods is that a Side Information (SI) has to be transmitted from the transmitter to the receiver to recover the original data, which results in some loss of throughput efficiency. Some recent efficient methods do not need any SI transmission [12]. The Active Constellation 
Extension (ACE) method proposed in [13] involves reducing PAPR by changing the constellation of the signal without changing the minimum distance. However, the performance of this method depends on the mapping level. Thus, it is not relevant for DVB-T2 system with QAM modulation up to 256 states and in the case of rotated constellation. The Tone Reservation (TR) method uses allocated subcarriers to generate additional information that minimizes the PAPR. An original classification representation for PAPR reduction techniques was studied and proposed in [3]. The TR method which is a sub-class of the adding signal technique will be our main concern. Thus, proposals for PAPR reduction in the case of DVB-T2 system will be restrained to methods issued from the TR concept.

This work was performed within the framework of the French regional Project DTTv2, which aimed at working on the improvements of DVB consortium standard: DVBT2 as well as the future mobile standard NGH (New Generation Handheld). This work includes the conception of an implementation and experimentation platform, allowing to study PAPR reduction in DVB-T2 context in real-time and conforming with industrial constraints.

This paper is organized as follows. Section 2 gives a brief description of the DVB-T2 system model and PAPR definition. The TR-based PAPR reduction techniques for DVB-T2 will be studied in Section 3. Also, we propose an iterative technique called "One Kernel One Peak" (OKOP) which is issued from the TR-gradient-based method. In Section 4, simulation results and comparison between the studied techniques will be presented. Section 5 describes the PAPR reduction block in the DTTv2 Experimentation Platform.

\section{DVB-T2 System Model and PAPR}

Some basic terms and the system model which include OFDM, DVB-T2 physical layer and PAPR, shall be presented first. Let us define the notations used throughout the paper. Time and frequency domain matrices are denoted by small and capital bold case letters, respectively. Scalars and vectors variables for the optimization equations are denoted by small and capital normal letters, respectively.

2.1. OFDM-Based DVB-T2 System. The OFDM signal is the sum of many orthogonally overlapped subchannels of equal bandwidth. In order to realize the spectrally overlapping sub-channels, the Inverse Fast Fourier Transform (IFFT) is employed at the OFDM transmitter. The base-band samples for OFDM symbol, with $N$ subcarriers, at the IFFT output are given by:

$$
x(t)=\frac{1}{\sqrt{N}} \sum_{k=0}^{N-1} X_{k} e^{j 2 \pi k t / T N}, \quad 0 \leqslant t \leqslant N T,
$$

where $T$ is the original complex symbol duration. In practice, we assume that only $N L$ equidistant samples of $x(t)$ are considered, where $L$ represents the oversampling factor. The DVB-T2 system employs optionally $1 \%$ of tones for PAPR reduction in TR context. The possible FFT sizes of a symbol in a T2-Frame are $N=1024,2048,4096,8192,16384$, and 32768 [1]. The associated possible modulation modes are QPSK, 16-QAM, 64-QAM, and 256-QAM.

2.2. PAPR Definition. Due to the statistical independence of carriers, the central-limit theorem holds and the complex time-domain samples of OFDM signals are approximately Gaussian distributed. This means that there could be some very high peaks present in the signal. Peak to Average Power Ratio (PAPR) is the most common term used in the literature to describe these temporal fluctuations of the signal. The PAPR defines the ratio of the signal's maximum instantaneous power to its mean power. The oversampled discrete-time OFDM symbol sample of $x(t)$ can be given by [14]:

$$
x_{n}=\frac{1}{\sqrt{N L}} \sum_{k=0}^{N-1} X_{k} e^{j 2 \pi n k / N L}, \quad \forall n \in[0, \ldots, N L-1],
$$

where $L$ is the oversampling factor. This factor must be large enough $(L \geq 4)$ to process all the continuous-time peaks and thus to better approximate the analog PAPR of the OFDM signal. Thus, the PAPR can be expressed as [14]:

$$
\begin{aligned}
\operatorname{PAPR}\{x(t)\} & \approx \operatorname{PAPR}\left\{x_{L}, L \geq 4\right\} \\
& =\frac{\max _{0 \leq n \leq N L-1}\left|x_{n}\right|^{2}}{\mathbb{E}\left|x_{L}\right|^{2}},
\end{aligned}
$$

where $\mathbf{x}_{\mathbf{L}}=\mathbf{Q}_{\mathrm{L}} \mathbf{X}_{\mathrm{L}}, \mathbf{X}_{\mathrm{L}}$ is the zero-padded vector of $\mathbf{X}$ by factor $L, \mathbb{E}\{\cdot\}$ denotes the expectation operation, and $\mathbf{Q}_{\mathrm{L}}$ is the inverse discrete Fourier transform matrix of size $N L$ scaled by $\sqrt{L}$. The PAPR reduction performance is evaluated using the Complementary Cumulative Distribution Function (CCDF). It is defined by the probability that the PAPR of the OFDM signal exceeds a given threshold $\gamma[15]$ :

$$
\mathrm{CCDF}_{\mathrm{PAPR}} x_{n}=\operatorname{Pr}\left[\operatorname{PAPR}\left\{x_{n}\right\}>\gamma\right] .
$$

\section{TR-Based PAPR Reduction Techniques}

3.1. Tone Reservation Methodology. In TR concept, the basic idea is to reserve some OFDM subcarriers called Peak Reduction Tones (PRT) for PAPR reduction. These reserved subcarriers do not carry any data information, and they are only used for reducing PAPR. This method restricts the data vector, and the peak reduction vector to lie in disjoint frequency subspaces. This formulation is distortionless and leads to very simple coding of the data subsymbols that are extracted from the received sequence by choosing the set of values at the receiver FFT output. Therefore, as natively included in the standard, this concept does not degrade the BER performance of the system, and thus can be categorized in downward compatible method [3]. The problem of computing the values for these reserved tones that minimize the PAPR can be formulated as a convex problem and can be solved exactly. The Second-Order Cone Program (SOCP) applied on unused subcarriers is described 




FIgURE 1: Tone Reservation method to reduce PAPR [12].

in [16]. This method has a high computational complexity. As consequence, suboptimal techniques which are able to converge faster than the optimal solution are the subject of this section.

3.2. Implementation Schemes for TR. In this paper, different implementation schemes for TR methods shall be discussed and compared. The idea is to reduce the PAPR of the signal $x=\operatorname{IFFT}(X)$ such that $\operatorname{PAPR}(x+c)<\operatorname{PAPR}(x)$, where $c$ represents the added peak reducing signal, as shown in Figure 1. Ideally the objective of reducing the peak of the combined signal $(x+c)$ should be attained while keeping the mean power constant or nearly unchanged. Mathematically, it can be expressed by:

$$
\mathbb{E}\left\{\|x+c\|^{2}\right\} \approx \mathbb{E}\left\{\|x\|^{2}\right\} .
$$

However, adding signal results in a mean power increase. The relative increase in the mean power $\Delta E$ is defined as [12]

$$
\Delta E=10 \log _{10} \frac{\mathbb{E}\left\{\|x+c\|^{2}\right\}}{\mathbb{E}\left\{\|x\|^{2}\right\}} .
$$

The aim should be to keep this $\Delta E$ as small as possible to meet the high power amplifier constraints. Increased mean power might drive the power amplifier into the saturation zone which results in nonlinearity and system performance degradation. We note that the phenomenon of decreased minimum distances in constellation due to increased mean power in the peak power control context is discussed in [17]. The $\Delta E$ must be upper bounded ensuring that individual component magnitude value cannot exceed a given value as indicated in [18],

$$
\Delta E<\gamma \mathrm{dB},
$$

which follows,

$$
\mathbb{E}\left\{\|x+c\|^{2}\right\} \leq \lambda \mathbb{E}\left\{\|x\|^{2}\right\},
$$

where $\lambda=10^{\gamma / 10}$ is a constant related to power amplifier characteristics.

3.2.1. TR-Clipping-Based Technique. This technique consists in applying a hard clipping to the input OFDM signal (see Figure 2) [19]. Then, the clipped signal is subtracted from the input signal to obtain the correction signal. After that, the correction signal is passed to an FFT/IFFT filter in order to comply with the TR concept. The clipped signal can be expressed as follows:

$$
y_{n}= \begin{cases}x_{n} & \text { if }\left|x_{n}\right| \leq A, \\ A e^{j \Phi_{n}} & \text { if }\left|x_{n}\right| \geq A,\end{cases}
$$

where $x_{n}=\left|x_{n}\right| e^{i \Phi_{n}}$ is the input signal, $y_{n}$ is the clipped signal, and $A$ is the clipping magnitude level. The correction signal is obtained from the differences between the samples of the useful multi-carrier signal $x_{n}$ and its clipped version $y_{n}$.

$$
c_{n}=x_{n}-y_{n}
$$

Figure 3 shows the peak-reducing signal generator block in the case of OFDM envelope clipping. To conform to the TR concept, only the values of the reserved tones at the PRT positions are kept; the others are reset to zero, thus:

$$
\begin{gathered}
C_{k}=\operatorname{FFT}\left(c_{n}\right), \\
\hat{C}_{k}= \begin{cases}C_{k} & \text { if } k \in \mathrm{PRT}, \\
0 & \text { if } k \notin \mathrm{PRT} .\end{cases}
\end{gathered}
$$

At each iteration, the algorithm updates the vector $X_{k}\left(X_{k}=\right.$ $\left.\operatorname{FFT}\left(x_{n}\right)\right)$ by adding to it the vector $\hat{C}_{k}$.

$$
X_{k}^{i+1}=X_{k}^{i}+\mu \hat{C}_{k},
$$

where $\mu$ is the step of the gradient method. Figure 4 shows the principle of adding signal technique for PAPR reduction with gradient-based method in frequency-domain issued from a classical clipping. The IB filtering block guarantees the downward compatibility by considering only frequency components of the correction signal at the PRT positions. Since this update rule is performed in the frequency domain, this algorithm can simply incorporate the necessary spectral constraint, by simply limiting the power of the reserved tones.

3.2.2. TR-Gradient-Based Technique. The time-domain gradient-based method for PAPR reduction is proposed in the DVB-T2 norm. This method associated with Tone Reservation concept was studied and proposed by TelladoMourelo in [12] and later defended by SAMSUNG for PAPR reduction scheme suitable for IEEE 802.16e. The principle of the gradient-based method is to iteratively cancel out the signal peaks by a set of impulse-like kernels. Reserved carriers are allocated according to predetermined carrier locations which are reserved carrier indices. After the IFFT, peak cancellation is operated to reduce PAPR by using a predetermined signal. The predetermined signal, or kernel, is generated by the reserved carriers.

The gradient algorithm is one of the good solutions to compute with low complexity. The basic idea of the gradient algorithm is come from clipping. Clipping the peak tone to the target clipping level can be interpreted as subtracting 


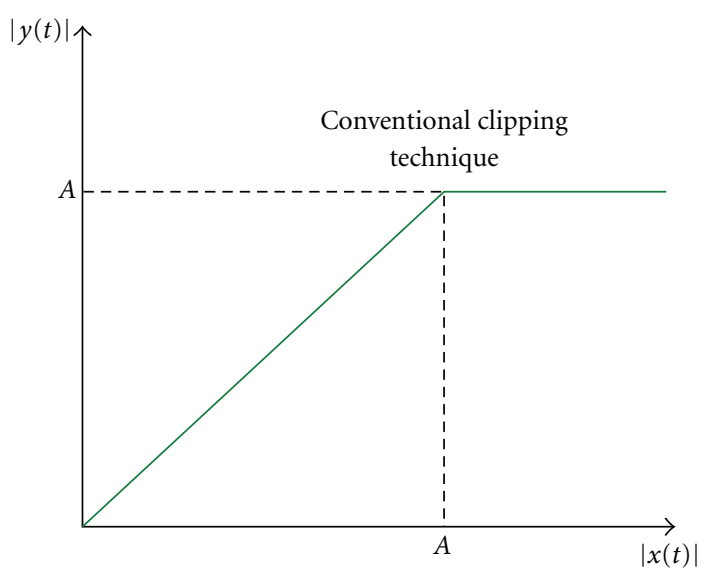

FIgURE 2: The classical amplitude clipping function.



FIGURE 3: The peak-reducing signal generator block in the case of OFDM envelope clipping.

impulse function from the peak tone in time domain. The conventional clipping technique can be formulated as an adding signal technique where its peak reducing signal is generated directly in time domain [20]. The principle of the TR gradient-based technique is presented in Figure 5. Despite of their low computational complexity, the gradientbased methods have the drawback of increasing the signal average power. In addition, this increase in the average power is dependent on the PAPR reduction gain.

(a) Impulse-Like Kernel Generation. During the first step, the kernel vector $p^{\| 2}$ is computed from the PRT and stored in memory during the initialization phase. For optimal performance, the generated kernel should be designed to be as close as possible to a discrete-time impulse. This way, every time the algorithm cancels a peak of $x$, no secondary peaks are generated at other locations. However, as in DVB-T2 the PRT are specified in advance, it is not possible to perfectly match with a discrete-time impulse. An optimum solution to generate the peak reduction kernel was studied in [12]; thus the kernel signal is defined as

$$
p^{\| 2}=\frac{\sqrt{N_{\mathrm{FFT}}}}{N_{\mathrm{PRT}}} \operatorname{IFFT}\left(\mathbf{1}_{\mathrm{PRT}}\right)
$$

where $N_{\text {FFT }}$ and $N_{\text {PRT }}$ indicate the FFT size and the number of PRT, respectively. The $\left(N_{\mathrm{FFT}}, 1\right)$ vector $\mathbf{1}_{\mathrm{PRT}}$ has $N_{\mathrm{PRT}}$ elements of ones at the positions corresponding to the reserved-carrier indices and has $\left(N_{\mathrm{FFT}}-N_{\mathrm{PRT}}\right)$ elements of zeros at the others.

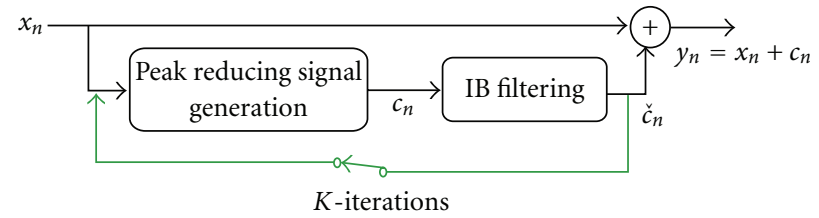

FIGURE 4: The principle of adding signal technique for PAPR reduction with gradient-based method in frequency-domain issued from a classical clipping.

(b) Peak Reduction Algorithm. The IFFT output $x$ is fed into the peak-cancellation block, and the peak position and value of $x$ are detected. Thus, for $n=0, \ldots, N_{\text {FFT }-1}$,

$$
\begin{gathered}
y_{i}=\max _{n}\left|x_{n}^{i}\right|, \\
m_{i}=\underset{n}{\operatorname{argmax}}\left|x_{n}^{i}\right|,
\end{gathered}
$$

where $x_{n}^{i}$ represents the $n$th element of the vector $x^{i} ; y_{i}$ and $m_{i}$ represent the maximum magnitude and the index of the detected peak in the $i$ th iteration, respectively. Then, in the second step of the algorithm, the reference kernel, generated by the reserved carriers corresponding to the current OFDM symbol, is circularly shifted to the peak position, scaled so that the power of the peak tone should be reduced to the desired target clipping level and phase rotated. The resulting kernel is subtracted from $x$ and the new PAPR is calculated. As the impulse-like function is designed with only the value in the reserved tone locations, adding the peak reducing signal to the data signal does not affect the value of OFDM symbol in frequency domain.

$$
x^{i+1}=x^{i}+\alpha_{i} p^{\| 2}\left(m_{i}\right)
$$

where

$$
\alpha_{i}=\frac{x_{m_{i}}^{i}}{y_{i}}\left(y_{i}-A\right),
$$

where $p^{\| 2}\left(m_{i}\right)$ denotes the kernel vector circularly shifted by $m_{i}$ and $A$ is the clipping magnitude level. In the third step, the PAPR of the resulting signal (after adding the peak reduction kernel to the useful data signal) is calculated. If the PAPR of the resulting signal satisfies the target PAPR level, this signal is transmitted. If not, the cancellation operation is repeated iteratively, until the number of iterations reaches the predetermined maximum iteration number. The peakcancellation method detects and removes only the maximum remaining peak in the time-domain per iteration. This method is simple and efficient in terms of peak regrowth control for the following iterations, at the expense of requiring a relatively large number of iterations. Alternatively, multiple peaks can be removed in a single iteration because the kernels can be linearly combined. However, this will increase 


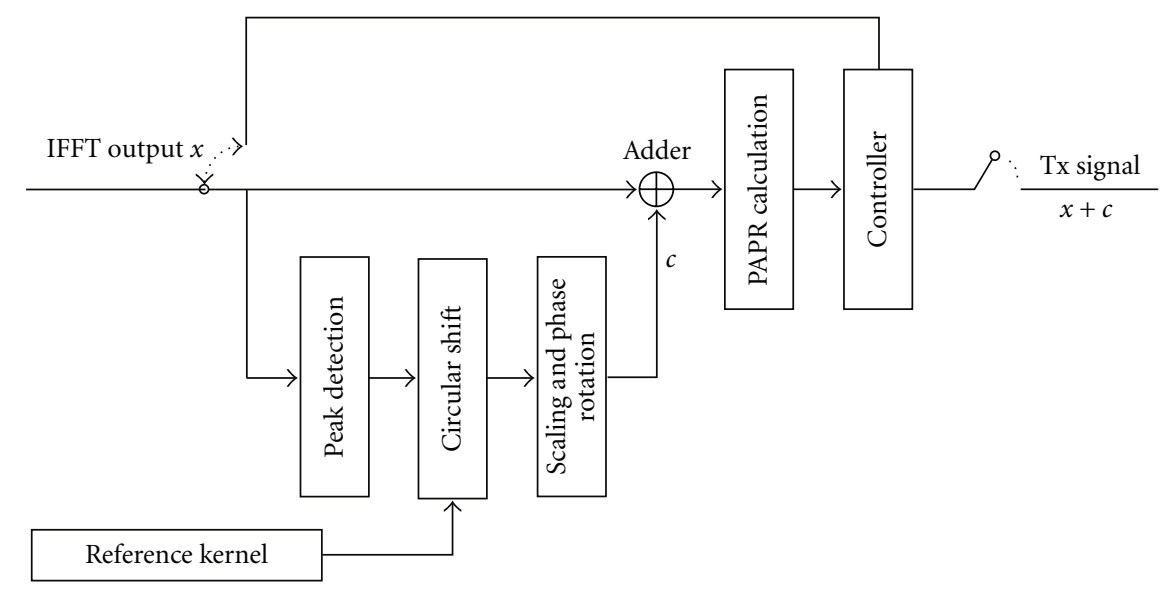

FIGURE 5: Block diagram of the peak-cancellation algorithm.

the number of computations per iteration. The transmitted signal after the $i$ th iteration of the simple method is given as

$$
\begin{aligned}
x^{i} & =x+\alpha_{1} p^{\| 2}\left(m_{1}\right)+\cdots+\alpha_{i} p^{\| 2}\left(m_{i}\right) \\
& =x+\sum_{k=1}^{i} \alpha_{k} p^{\| 2}\left(m_{k}\right) .
\end{aligned}
$$

3.2.3. Proposed Method (TR-OKOP). The same energy is added to each reserved subcarrier at each iteration of the TR algorithm. The difficulty resides in how we can predict the evolution of the vectorial sum on each subcarrier. Controlling the power of a reserved subcarrier implies passing to frequency domain or maintaining in memory the information on the amplitude and phase of each subcarrier at each iteration of the algorithm. The DVB-T2 system is defined with a large number of subcarriers (up to 32768 subcarriers). The number of reserved subcarriers for the $16 \mathrm{~K}$ and the $32 \mathrm{~K}$ mode are 144 and 288, respectively. Thus, the method that we propose, called "One Kernel One Peak" $(\mathrm{OKOP})$, consists in distributing the reserved subcarriers into groups. Then one impulse-like kernel signal is generated from each group of the reserved subcarriers (see Figure 6). The original idea here consists on using one kernel to reduce one peak. A simple modification on the TR-Gradient-based algorithm permits the implementation of this technique. The modification concerns the impulse-like kernel generation part of the algorithm presented in the previous section. It offers the capability to control independently the power associated to each group of PRT. This means that instead of using the same reference signal at each iteration, a unique correction signal (generated from a specific group of subcarriers) is added to the useful signal. Thus, there is as much iteration as correction signals during one pass. Also, at each pass, the PRT are used only one time.

\section{Simulation Results and Comparison}

The simulation model is designed to match with the DVBT2 standard. The number of PRT is $R=10,18,36,72$,



FIGURE 6: Correction signal generation from PRT with the TROKOP algorithm.

144, or 288, while the FFT size is, respectively, $N=1024$, $2048,4096,8192,16384$, or 32768 , with the number of subcarriers in use, $K=853,1705,3409,6913,13921$, or 27841 , respectively. It should be noted that the power of the correction carriers should not exceed the power spectrum mask specified for DVB-T2 by more than $10 \mathrm{~dB}$. The performance of the TR-based methods is compared in terms of PAPR reduction capability, computational complexity and system interference (BER). Also, the power spectral density (PSD) presentations are provided to evaluate the impact of applying the TR methods on the power spectrum mask.

4.1. PAPR Reduction Performance. Simulation results using Matlab (see Figures 7 and 8) show that both algorithms, TR-Clipping and TR-Gradient, have equivalent performance in term of PAPR reduction gain. However, the TR-Gradient method is less complex (in term of number of operations) than the TR-Clipping because all the treatments are provided in the time domain. It does not include an IB and OOB filter since the correction signal is generated directly from the reserved tones. The advantage of the TR-Clipping technique is that the update rule is performed in the frequency domain. 


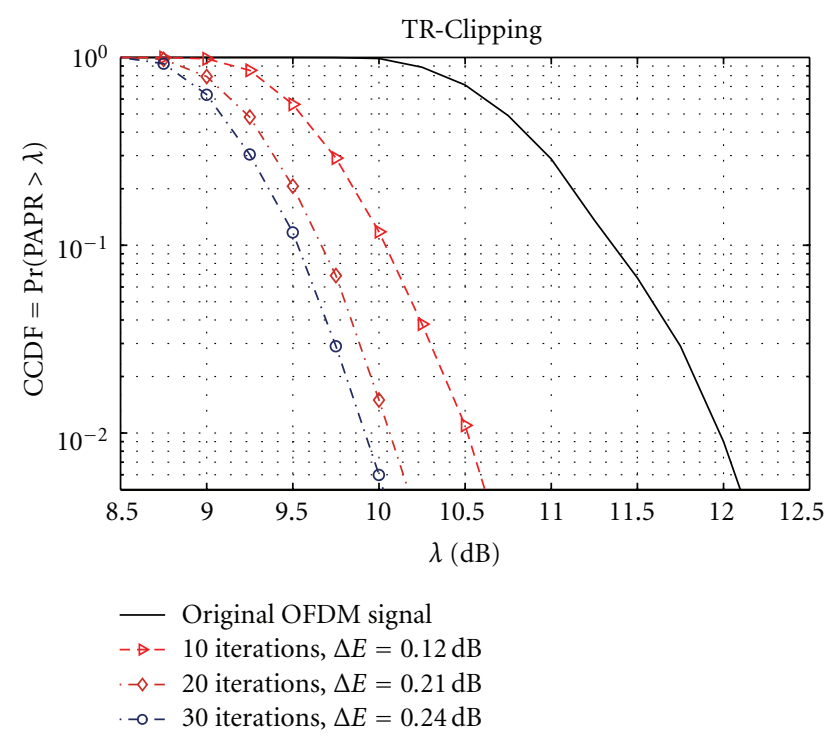

FIgure 7: PAPR CCDF for different iterations' numbers, $L=4$, DVB-T2 parameters, $N=32 \mathrm{~K}, 256 \mathrm{QAM}$ with the TR-Clipping method.

Therefore, this algorithm can simply incorporate the necessary spectral constraint, by simply limiting the power of the reserved tones. The performance of the proposed TR-OKOP technique is compared to the TR-Clipping and TR-Gradient in Figure 9. With the TR-OKOP, the PRTs are divided into 36 groups. Thus, a correction signal (kernel) is generated using 8 subcarriers. The term "pass" in Figure 9 refers to the use of all the reserved subcarriers for PAPR reduction (288 PRTs in the $32 \mathrm{~K}$ mode) or all the generated correction signals (36 kernels) only once. Thus, at each pass, 36 peaks are reduced using 36 correction signals. The proposed algorithm has the same performance in term of PAPR reduction compared to the other algorithms. Its advantage lies in its capability to control independently the power associated to each group of PRTs.

4.2. Complexity Analysis. In this section, we evaluate the complexity performance of the different implementation schemes for TR methods described in Section 3. Only the runtime complexity in term of the number of operations is considered and the complexity of the initialization stage is omitted since it occurs only once.

4.2.1. TR-Clipping-Based Technique. Let us start by evaluating the complexity of the algorithm in the loop. As discussed in a previous section, this algorithm evaluates the correction signal $c_{n}$. Then, the correction signal is passed to a filter based on FFT/IFFT pair in order to comply with the TR concept. The complexity of calculating $c_{n}$ is very low compared to the complexity of calculating the filtered correction signal $\breve{c}_{n}$ and can be omitted. Therefore, the complexity of the algorithm is approximated as $O\left(2 \times N L \log _{2} N L\right)$.

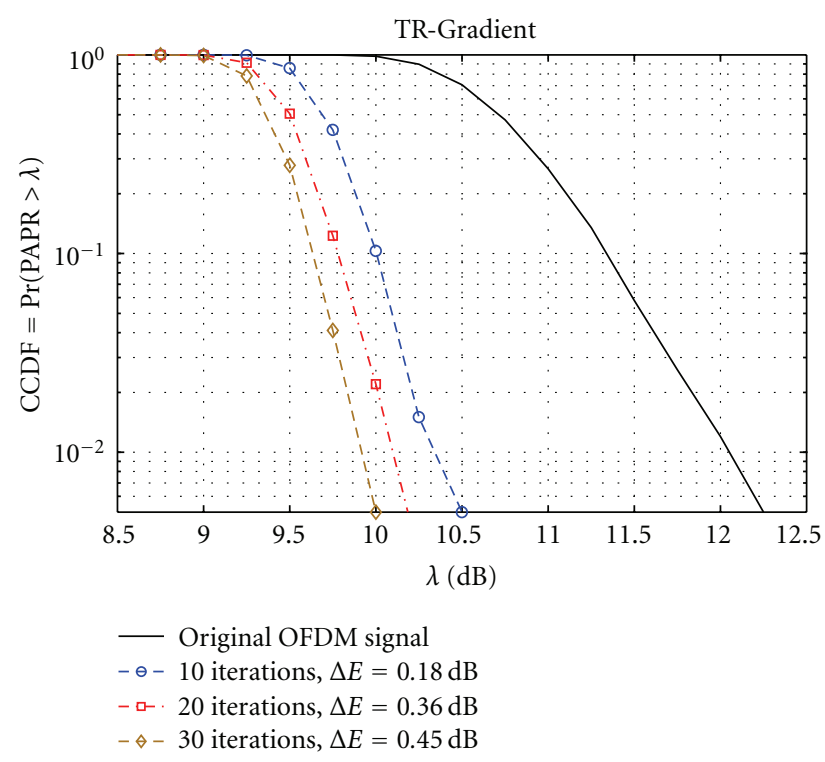

FIGURE 8: PAPR CCDF for different iterations' numbers, $L=4$, DVB-T2 parameters, $N=32 \mathrm{~K}, 256$ QAM with the TR gradientbased method.

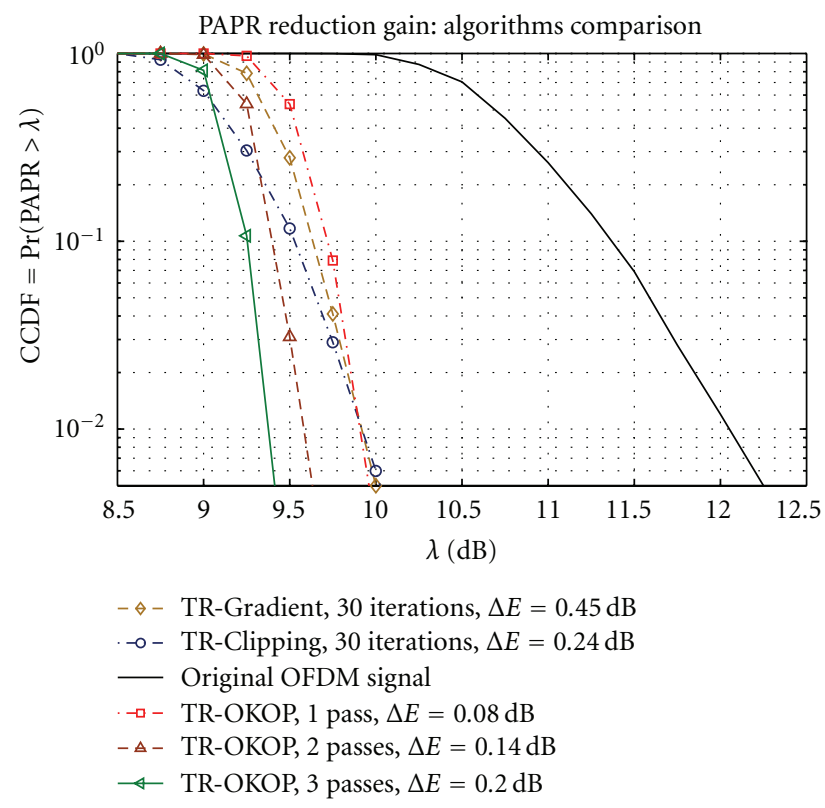

FIGURE 9: Comparison between the use of different PAPR reduction methods with DVB-T2 parameters, $L=4, N=32 \mathrm{~K}, 256$ QAM.

4.2.2. TR-Gradient-Based Technique. This technique operates in time domain. The correction signal (reference kernel) is computed from the PRT and stored in memory during the initialization phase. The other steps consist in circularly shifting the reference kernel to the peak position, scaled and phase rotated. The complexity of calculating the time domain samples of the peak-canceling signal from the reference kernel is $O(N L)$. 


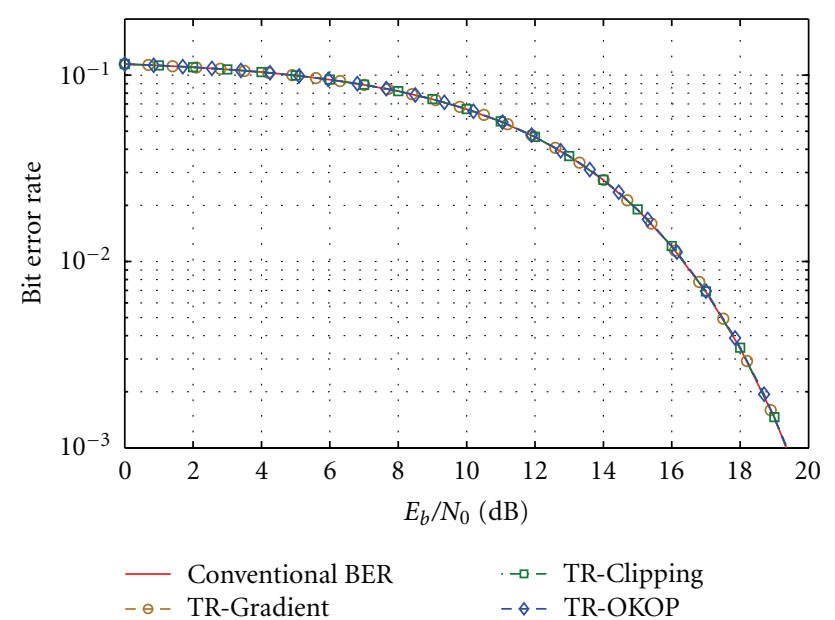

Figure 10: BER versus $E_{b} / N_{0}$ for the three implementation schemes of TR methods with DVB-T2 parameters, $N=32 \mathrm{~K}, 256$ QAM.

4.2.3. Proposed TR-OKOP Method. The proposed method computes a reference kernel from a group of PRTs at each iteration. This means that an IFFT operation is applied at each iteration. As for the TR-Gradient-based method, the other steps consist on circularly shifting the reference kernel to the peak position, scaled and phase rotated. Therefore, the complexity of calculating the time domain samples of the peak-canceling signal from the reference kernel is $O\left(N L \log _{2} N L\right)$.

The Gradient-based technique has the advantage in term of complexity over the TR-Clipping one. The complexity of the proposed TR-OKOP technique is higher than that of the Gradient-based one and lower than that of the TR-Clipping one. The advantage of the proposed technique is that the PRT are used only once during one algorithm pass. This allows an easier control of the power variation on each reserved subcarrier.

4.3. $I B$ and $O O B$ Interference Analysis. As explained in a previous section, all the TR-based PAPR reduction methods do not affect the BER performance. The TR-Gradient and the TR-OKOP techniques create the correction signal from reserved carriers. Thus, the data carriers are not affected. For the TR-clipping technique, the generated correction signal passes through an FFT/IFFT filter in order to respect the TR concept. Therefore, it is evident in Figure 10 that the three methods match the conventional BER performance curve thus proving the hypothesis that out of useful band tones do not create IB interference and thus no BER degradation takes place. It should be noted that BER calculations are performed for useful carriers only.

The OOB distortions are nullified thanks to the FFT/IFFT filter for the TR-Clipping technique. Figure 11 shows the PSD of an OFDM signal before and after applying the TR-Gradient PAPR reduction technique. We observe that the power level of the PRT can exceed that of the useful signal by more than $10 \mathrm{~dB}$. In Figure 12, the proposed algorithm TR-OKOP was applied. In this case, the power

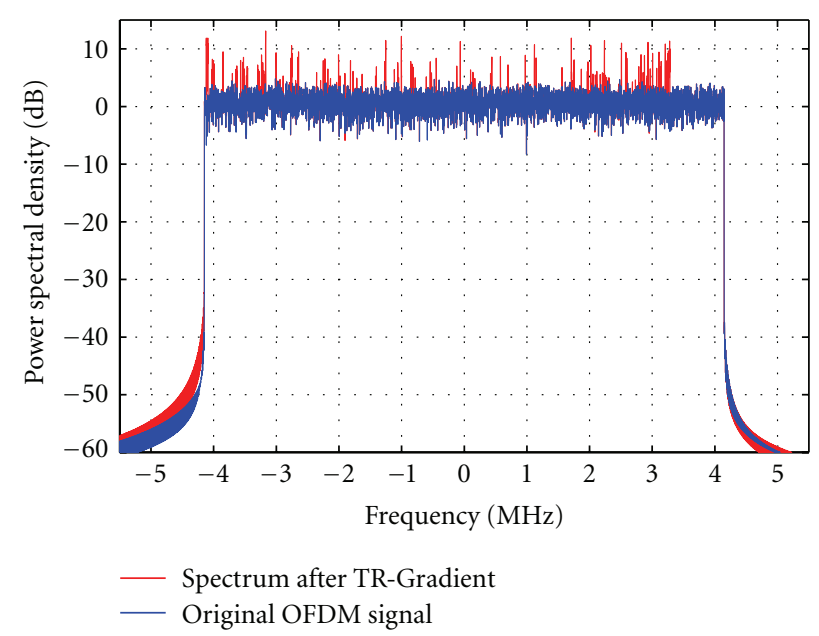

Figure 11: Power spectral density of an OFDM signal (DVB-T2 parameters, $L=4, N=32 \mathrm{~K}, 256 \mathrm{QAM}$ ) after applying the TRGradient PAPR reduction technique.

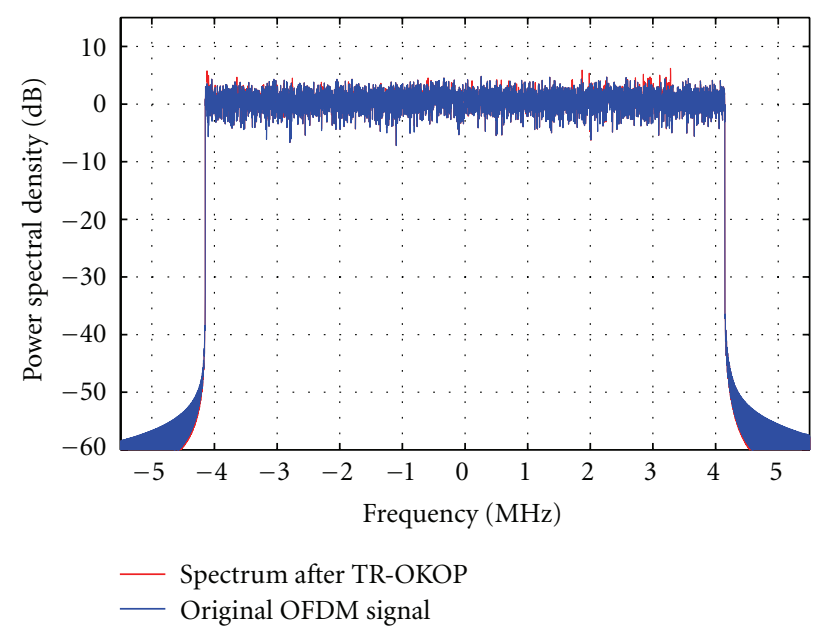

Figure 12: Power spectral density of an OFDM signal (DVB-T2 parameters, $L=4, N=32 \mathrm{~K}, 256 \mathrm{QAM}$ ) after applying the proposed TR-OKOP PAPR reduction technique.

spectrum specifications are respected. Also, Figure 9 shows that both algorithms achieve the same PAPR reduction gain for different values of power variation. Also, the mean power variation in the case of TR-OKOP is lower than that of the TR-Gradient.

Table 1 summarizes the performance comparison between the three TR-based PAPR reduction methods in terms of PAPR reduction gain, mean power variation, complexity, and spectrum control capability. In Table 1, the sign "++" in the complexity line signifies that the method has a low complexity.

\section{PAPR Reduction Algorithm Implementation}

5.1. DTTv2 Platform. DTTv2 platform is an industrial implementation of the DVB-T2 standard. It processes input stream (which can be for instance an encoded video stream) 


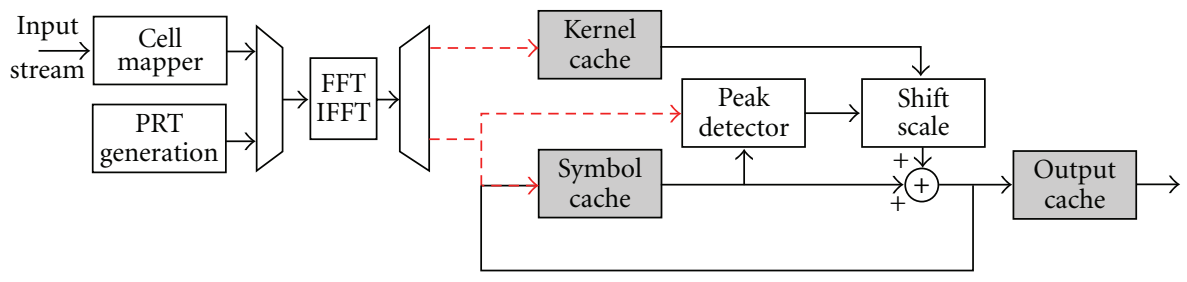

FIgURE 13: FPGA implementation of the PAPR reduction block in the DTTv2 modulator.

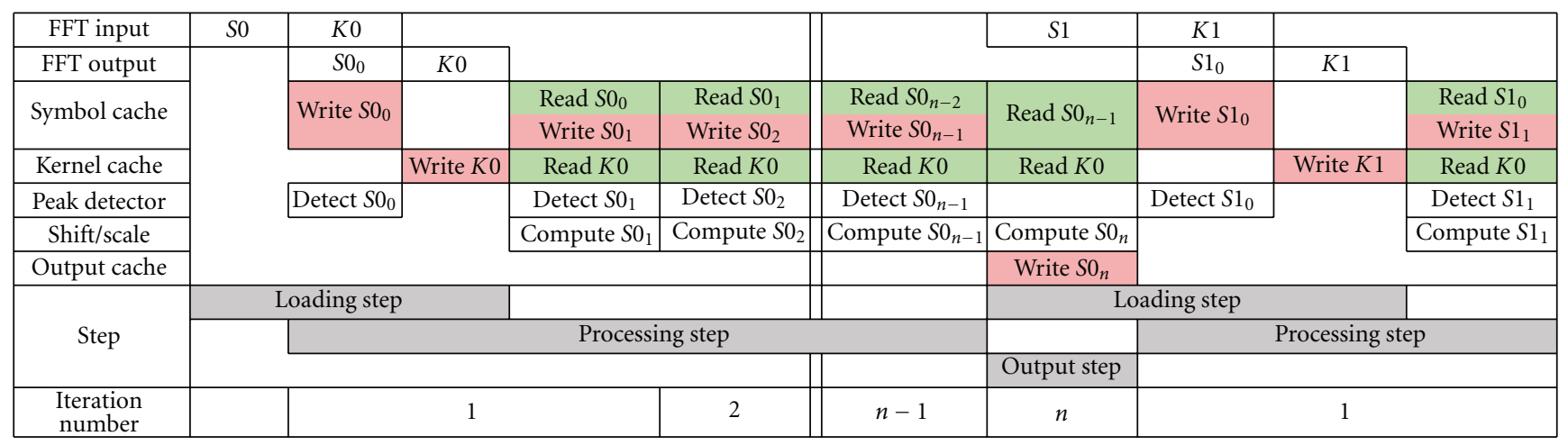

FIGURE 14: Block utilization during the TR-Gradient processing.

TABle 1: Performance comparison of TR-based PAPR reduction methods.

\begin{tabular}{lccc}
\hline & TR-Gradient & TR-Clipping & TR-OKOP \\
\hline$\Delta$ PAPR & ++ & ++ & ++ \\
$\Delta E$ & - & + & + \\
Complexity & ++ & - & + \\
Spectrum control & - & ++ & + \\
\hline
\end{tabular}

and generates a compliant DVB-T2 RF signal. Most of the computation is done using a Field Programmable Gate Array (FPGA), with the help of software when no real-time processing is required. After channel coding (which includes Forward Error Correction, interleaving and mapping on constellation), OFDM symbols are assembled by adding pilot carriers, including PRT when PAPR reduction using TR is enabled (see Figure 15). PAPR reduction block implements the TR-Gradient algorithm, as defined in DVB-T2 standard [18]. Thus, it operates in time domain after IFFT. A CCDF estimator is placed after up-sampling filters, to monitor the performance. Finally the signal is converted to analog IF and then up-converted to RF, in the UHF-VHF bands.

5.2. PAPR Reduction in the DTTv2 Platform. This section describes the TR-Gradient-based PAPR reduction block as implemented in the DTTv2 platform (see Figure 13). The design choice was to insert the algorithm within the existing modulation processing blocks, allowing to share and optimize hardware resources usage.

5.2.1. Main Blocks Description. The first block Cell Mapper aggregates QAM-mapped data and OFDM pilot carriers



FIGURE 15: PAPR reduction block in the DTTv2 hardware platform.

(including PRT) to form a frequency domain symbol that is then processed by an IFFT to obtain a time domain symbol. Three memory-caches are used: "Kernel cache" that is used to save the current kernel, "Symbol cache" used to store initial and iterations results, and "Output cache" that is used to store the symbol after the final iteration that has been completed. The Peak-detector unit is in charge of detecting and storing peaks locations, that will be then used by the Shift-Scale unit to compute the appropriate peak-canceling signal.

5.2.2. Processing Description. For each processed symbol, several separate steps can be distinguished.

(i) Loading Step. A kernel is computed for each symbol to save memory; during symbols generation by Cell Mapper, PRT locations are saved and are later used to compute the kernel. At the end of this step, symbol cache and kernel cache are filled with corresponding symbol and kernel. While the symbol cache is written, the symbol is also processed for peak 
detection. This specific data flow is identified with red dotted path on Figure 13.

(ii) Processing Step. During each iteration, the Shift-Scale unit computes a peak-canceling symbol that is added to the symbol. The symbol is then written to its cache memory while remaining peaks are being localized at the same time to prepare the next iteration.

(iii) Output Step. When end-criteria are matched (the maximum number of iteration has been reached, PAPR is below the target, or limits condition on $\Delta E$ requires to stop iterations), the symbol is written to the output cache. This one adds cyclic prefix and streams the symbol to the next block.

(iv) Pipelining. Figure 14 shows the block usage during the processing and the associated steps. Some pipelinings were applied when it was possible; however, the IFFT output is bit-reversed and thus a cycle is lost to rewrite the symbols in natural order. Overall throughput can be improved by using additional memory for that purpose.

5.2.3. Performance. The maximum number of iterations is limited by the available time between OFDM symbols generation and cannot be easily improved. However, the possible number of canceled peaks can be increased by removing several peaks in one iteration. This mainly depends on the ability of the kernel cache memory to support multiple read operation, as the complexity of the additionally needed Shift-Scale units can be considered as marginal. By reusing already existing operators in the design (IFFT and a large amount of cache memory), this architecture implements DVB-T2 TR-Gradient PAPR reduction into a FPGA with a low hardware cost overhead, compared to the complexity of DVB-T2 processing in general.

\section{Conclusion}

Robustness and efficiency within DVB-T2's transmission system are further increased by new technologies such as PAPR reduction. In this paper, the performance of two TRbased PAPR reduction methods, gradient and clipping, is evaluated. Also, an iterative method called "One Kernel One Peak" (OKOP) is proposed. It offers the advantage of controlling the mean power increase of the reserved carriers. The performance of these methods is compared in terms of PAPR reduction capability, computational complexity and system interference (BER). Simulation results based on CCDF curves, using the DVB-T2 parameters, show that these methods offer an equivalent performance in term of PAPR gain. They provide a PAPR reduction gain of about $2 \mathrm{~dB}$ when only $1 \%$ of subcarriers is used without BER degradation. Thus, the data throughput is not reduced significantly. The advantage of the proposed TR-OKOP method is that the power of the correction carriers could be controlled more easily than in the case of the TRGradient method. Thus, the magnitude of the PRT could be set equal to the data subcarrier magnitude level. Also, the implementation of the TR-Gradient PAPR reduction algorithm in the DVB-T2 modulator was described.

\section{Acknowledgment}

The authors wish to thank the Pôle Images \& Réseaux for the financial support of this work.

\section{References}

[1] ETSI, "Digital Video Broadcasting (DVB); Implementation guidelines for a second digital terrestrial television broadcasting system (DVBT2)," ETSI TR 102831 v0.9.6, January 2009.

[2] S. H. Han and J. H. Lee, "An overview of peak-to-average power ratio reduction techniques for multicarrier transmission," IEEE Wireless Communications, vol. 12, no. 2, pp. 56-65, 2005.

[3] Y. Louët and J. Palicot, "A classification of methods for efficient power amplification of signals," Annals of Telecommunications, vol. 63, no. 7-8, pp. 351-368, 2008.

[4] R. O'Neill and L. B. Lopes, "Envelope variations and spectral splatter in clipped multicarrier signals," in Proceedings of the 6th IEEE International Symposium on Personal, Indoor and Mobile Radio Communications (PIMRC '95), pp. 71-75, Toronto, Canada, September 1995.

[5] J. Armstrong, "Peak-to-average power reduction for OFDM by repeated clipping and frequency domain filtering," Electronics Letters, vol. 38, no. 5, pp. 246-247, 2002.

[6] X. Li and L. J. Cimini Jr., "Effects of clipping and filtering on the performance of OFDM," IEEE Communications Letters, vol. 2, no. 5, pp. 131-133, 1998.

[7] A. E. Jones, T. A. Wilkinson, and S. K. Barton, "Block coding scheme for reduction of peak to mean envelope power ratio of multicarrier transmission schemes," Electronics Letters, vol. 30, no. 25, pp. 2098-2099, 1994.

[8] J. A. Davis and J. Jedwab, "Peak-to-mean power control in OFDM, Golay complementary sequences, and Reed-Muller codes," IEEE Transactions on Information Theory, vol. 45, no. 7, pp. 2397-2417, 1999.

[9] S. H. Müller and J. B. Huber, "OFDM with reduced peakto-average power ratio by optimum combination of partial transmit sequences," Electronics Letters, vol. 33, no. 5, pp. 368369, 1997.

[10] R. W. Bäuml, R. F. H. Fischer, and J. B. Huber, "Reducing the peak-to-average power ratio of multicarrier modulation by selected mapping," Electronics Letters, vol. 32, no. 22, pp. 2056-2057, 1996.

[11] A. D. S. Jayalath and C. Tellambura, "Reducing the peak-toaverage power ratio of orthogonal frequency division multiplexing signal through bit or symbol interleaving," Electronics Letters, vol. 36, no. 13, pp. 1161-1163, 2000.

[12] J. Tellado-Mourelo, Peak to average power reduction for multicarrier modulation, Ph.D. thesis, Stanford University, Stanford, Calif, USA, September 1999.

[13] B. S. Krongold and D. L. Jones, "PAR reduction in OFDM via active constellation extension," IEEE Transactions on Broadcasting, vol. 49, no. 3, pp. 258-268, 2003.

[14] M. Sharif, M. Gharavi-Alkhansari, and B. H. Khalaj, "On the peak-to-average power of OFDM signals based on oversampling," IEEE Transactions on Communications, vol. 51, no. 1, pp. 72-78, 2003. 
[15] R. Van Nee and R. Prasad, OFDM for Wireless Multimedia Communications, Artech House, Boston, Mass, USA, 2000.

[16] S. Zabre, J. Palicot, Y. Louët, and C. Lereau, "SOCP approach for OFDM peak-to-average power ratio reduction in the signal adding context," in Proceedings of the 6th IEEE International Symposium on Signal Processing and Information Technology (ISSPIT '06), pp. 834-839, Vancouver, Canada, August 2006.

[17] R. Baxely, Analyzing selected mapping for peak to average power reduction in OFDM, M.S. thesis, Georgia Institute of Technology, May 2005.

[18] ETSI, "Digital Video Broadcasting (DVB); Frame structure channel coding and modulation for a second generation digital terrestrial television broadcasting system (DVB-T2)," ETSI EN 302755 v1.2.0c, July 2009.

[19] S. Litsyn, Peak Power Control in Multicarrier Communications, Cambridge University Press, Cambridge, UK, 2007.

[20] D. Guel and J. Palicot, "Clipping formulated as an adding signal technique for OFDM peak power reduction," in Proceedings of the 69th IEEE Vehicular Technology Conference (VTC '09), Barcelona, Spain, April 2009. 

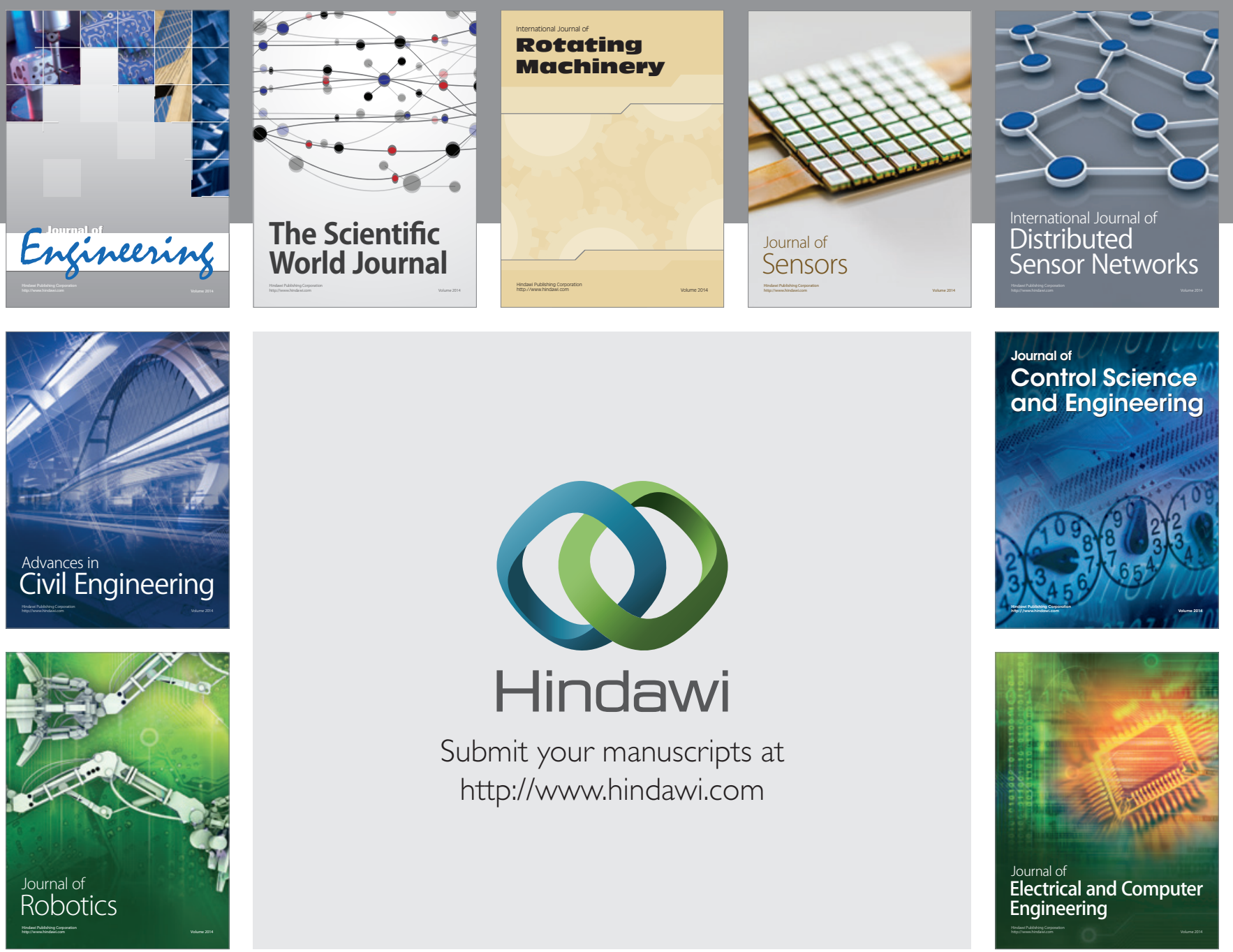

Submit your manuscripts at

http://www.hindawi.com
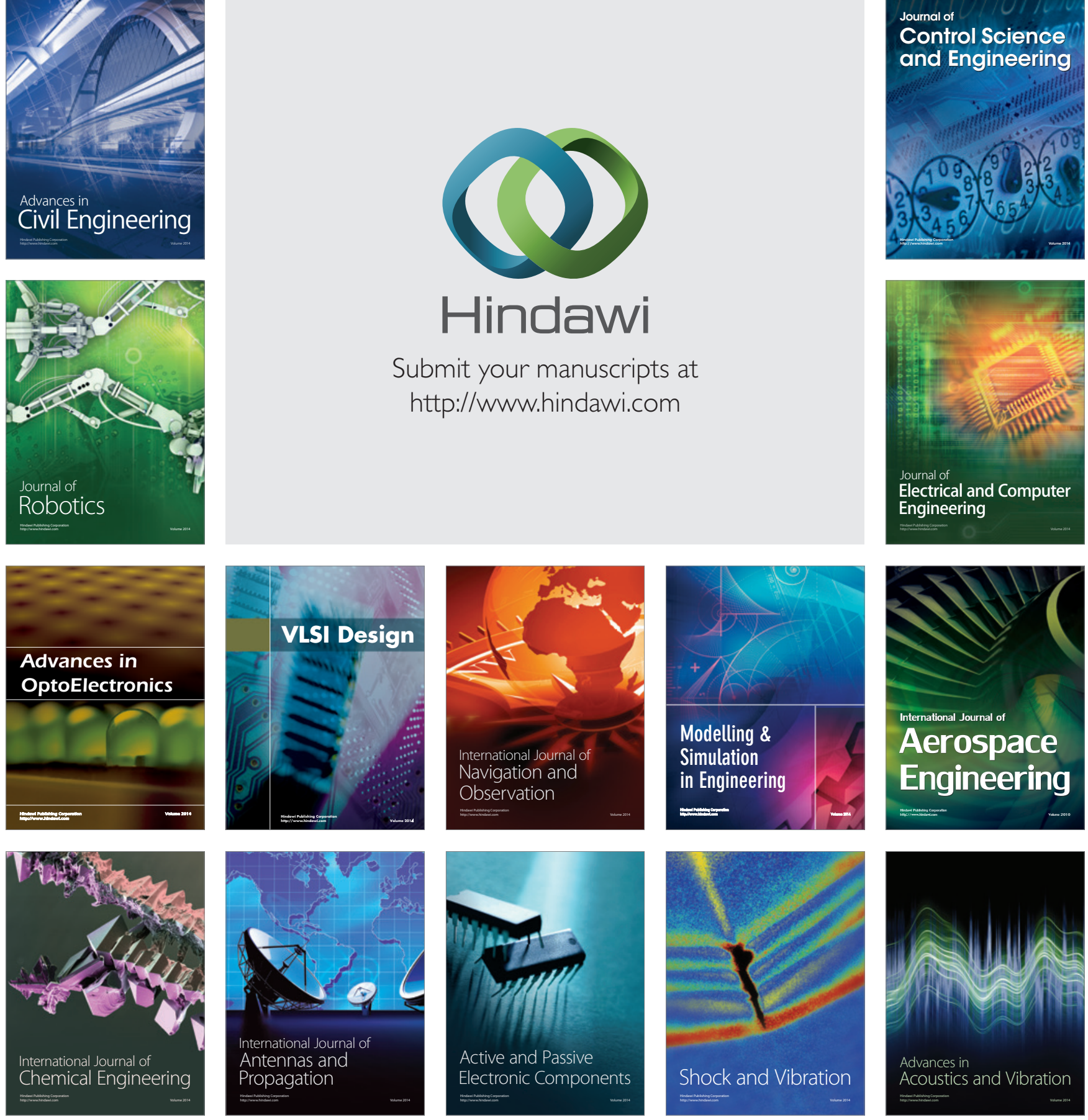